\title{
La responsabilidad social corporativa de la TV local: estudio de caso*
}

\author{
Corporate Social Responsibility of Local TV: A Case Study \\ Ana Criollo-Uyaguari \\ Ingeniera comercial, Universidad Politécnica Salesiana, \\ Quito-Ecuador, acriollou@est.ups.edu.ec \\ Andrea Feijóo-Valarezo \\ Ingeniera comercial, Universidad Politécnica Salesiana, \\ Quito-Ecuador, afeijoov@est.ups.edu.ec \\ Angel Torres-Toukoumidis \\ Doctor en comunicación, Universidad Politécnica Salesiana, \\ Quito-Ecuador, atorrest@ups.edu.ec
}

\begin{abstract}
Cómo citar / How to cite
Criollo-Uyaguari, A.; Feijóo-Valarezo, A.; Torres-Toukoumidis, A. (2020). La responsabilidad social corporativa de la TV local: estudio de caso. Revista CEA, v. 6, n. 12, 47-68. https://doi.org/10.22430/24223182.1583
\end{abstract}

Recibido: 23 de enero de 2020

Aceptado: 21 de mayo de 2020

\section{Resumen}

La responsabilidad social corporativa en la industria de los medios de comunicación se prescribe como una temática actual de debate en la comunidad académica, por ende, esta investigación pretende presentar la realidad de un canal de TV local de Ecuador, con base en los problemas in situ enfocados a la responsabilidad social, según la percepción de agentes internos y externos. Para ello, se aplicó un estudio cuanti-cualitativo de enfoque deductivo, combinando el alcance descriptivo con el diseño de investigación-acción, a través de encuestas a los agentes externos y entrevistas en profundidad al personal interno, generándose un plan de acción concreto para mejorar la responsabilidad social en los medios locales. Los resultados demuestran que, en general y según lo comentado por el personal interno de los medios locales, se maneja un buen ambiente laboral y los empleados se expresan satisfechos por el soporte emocional y social que reciben. Sin embargo, se necesita impulsar más acciones orientadas al cuidado medioambiental; por su parte, los agentes externos conocidos como clientes, proveedores y entes públicos solicitan que el medio busque optimizar los procesos de atención a sus necesidades; por el contrario, la mayoría reitera la importancia de la confianza en la interacción para generar empatía y fortalecimiento de lazos comerciales. De allí, se puede concluir que todo medio de comunicación, independientemente de la magnitud y audiencia, necesita sistematizar los procesos de responsabilidad social, con el fin de proveer un desarrollo sostenible a corto, mediano y largo plazo.

Palabras clave: responsabilidad social, medios de comunicación de masas, Ecuador.

\footnotetext{
* Este artículo se deriva del proyecto titulado «La responsabilidad social corporativa de la TV local: estudio de caso» y ha sido financiado con recursos propios.
} 
Clasificación JEL: L82; M14.

\begin{abstract}
Corporate social responsibility in the mass media industry is a current topic in the debate of the academic community. Therefore, this study aims to describe the reality of a local Ecuadorian TV station based on the problems related to social responsibility found on site as perceived by internal and external stakeholders. For that purpose, we applied a deductive quanti-qualitative approach that combined descriptive research and action research by means of surveys answered by external stakeholders and in-depth interviews with employees. As a result, we created a concrete action plan to improve the corporate social responsibility of local media. The results show that, in general and as commented by the employees of local media, their organizations have a good work environment, and they are satisfied with the emotional and social support they receive. Nevertheless, such companies should promote more actions to protect the environment. The media should also optimize their processes to address the needs of external stakeholders (that is, customers, suppliers, and public organizations). Most respondents confirmed the importance of trust in the interaction to develop empathy and strengthen commercial relations. As a result, we can conclude that any kind of medium, regardless of its size and audience, should systematize its social responsibility processes in order to provide sustainable development in the short, medium, and long term.
\end{abstract}

Keywords: Social responsibility, mass media, Ecuador.

JEL classification: L82; M14.

\title{
1. INTRODUCCIÓN
}

Durante los últimos años se han venido generando debates, tanto en el ámbito académico como profesional, sobre el papel que desempeñan las empresas ante la sociedad, con la finalidad de que puedan implementar acciones que vayan más allá de la búsqueda de beneficios y rentabilidad para sus accionistas (Giarrizzo \& Maceri, 2019). De hecho, la responsabilidad social corporativa es esencialmente una necesidad estratégica, donde las empresas deciden, de manera voluntaria, pasar de las buenas intenciones a las buenas acciones, en las cuales se valora el impacto positivo que se genera ante las comunidades, que al final son quienes la evalúan (Park et al., 2015; Dhanesh, 2020). La relación que existente entre la responsabilidad social corporativa y el éxito de una empresa se basa principalmente en crear efectos positivos entre los grupos de interés, tanto internos como externos, en donde sin duda el mayor protagonismo lo tienen los clientes y los consumidores (Wilson, 2012). También se ha evidenciado que dentro de este sector la responsabilidad social es una terminología empleada hacia el contenido y libertad de expresión (Zyglidopoulos et al., 2011), por lo que este estudio busca presentar una diferenciación con aquella denominada como responsabilidad social corporativa, donde se definen varios principios básicos, y que está orientada hacia la perspectiva de la industria mediática, identificada como un modelo de gestión empresarial.

En efecto, como López Salazar (2013) expresa, se ha llegado a demostrar que las personas prefieren las organizaciones que fomentan prácticas y criterios de sostenibilidad en los servicios y bienes que ofertan, y que abren paso a la responsabilidad social corporativa que, si bien se evidencia como una prioridad, aún se encuentra en una fase de desarrollo, análisis y exploración. Complementariamente, Men (2014) admite la incidencia de la responsabilidad social corporativa en los diversos grupos de interés, la misma que forma parte de los intangibles que han experimentado un mayor auge dentro de la gestión empresarial, coadyuvando a que la organización perciba una mejor reputación, incremento de la preferencia del público, e internamente pueda atraer y mantener un equipo humano comprometido (Rostro Hernández \& Solís Hernández, 2015). 
Por lo anterior, se procede a realizar un estudio enfocado en una empresa de comunicación, que forma parte del sector televisivo. El objetivo general es analizar la aplicación de la responsabilidad social corporativa en Unsion TV, derivando los siguientes objetivos específicos: [1] diagnosticar la aplicación de la responsabilidad social corporativa, con base en el personal interno de Unsion TV; [2] evaluar la responsabilidad social corporativa en los grupos externos de Unsion TV; y, por último, [3] sugerir un plan de acción orientado a una eficiente responsabilidad social corporativa en el medio de comunicación local.

\section{MARCO TEÓRICO}

La responsabilidad social corporativa tiene como finalidad proveer de eficiencia a la gestión empresarial (Fernández Lombao \& Campos Freire, 2013), mediante el compromiso voluntario con el desarrollo de la sociedad desde su composición social y un comportamiento responsable hacia las personas y grupos con quienes interactúa (Lizcano, 2004; Cho \& Tsang, 2020), englobando aspectos de las responsabilidades económica, ética - de la cual se derivan los compromisos legales-y hacia el medio ambiente, del respeto a los derechos humanos, laborales y sociales, pretendiendo así gestionar acciones orientadas por los principios de transparencia, materialidad, verificabilidad, visión amplia, mejora continua y naturaleza social de la organización (Barrio Fraile, 2019). Así también, la responsabilidad social corporativa incluye el compromiso de proporcionar sus servicios o bienes de una manera justa, sostenible y con responsabilidad (Fernández Lombao \& Campos Freire, 2013). Se puede decir que es una noción moderna basada sobre todo en un enfoque de concienciación sobre los impactos, que plantea una actividad estratégica que afecte a todo el conjunto de la organización y a todos sus grupos de interés, y no solo a uno en concreto como lo es una acción social (HernándezPerlines \& Sánchez-Infantes 2016).

Como se mencionó anteriormente, existen grupos con quienes la empresa se relaciona y que se denominan como stakeholders, siendo estos cualquier grupo o individuo que pueda afectar o ser afectado por el logro de los objetivos de la empresa (Weitzner \& Deutsch, 2019). De aquí en adelante, la visión de la empresa, que anteriormente se enfocaba en la producción y en la parte gerencial, pasa a redefinirse bajo una perspectiva donde los líderes ya no deben dirigirse en sus actividades solo en busca de un beneficio máximo para los accionistas o ser instrumento para la obtención de alcances económicos (Bardos et al., 2020), sino que deben tener por meta velar, satisfacer y cumplir, en lo que más esté a su alcance, los diferentes intereses y objetivos de los grupos que, de alguna manera, se involucran o tienen una relación con la empresa. Incluso la responsabilidad social corporativa de grandes transnacionales tenía la necesidad de reescribir su pasado y no solo responder a las exigencias del presente (Gil Sánchez, 2018). No obstante, es imposible comprender la responsabilidad corporativa y su gestión, sin considerar el nuevo paradigma de empresa formado por todo el conjunto de stakeholders compuesto por empleados, consumidores, administraciones públicas, proveedores y la sociedad (Barrio \& Enrique, 2018), que al final serán los que decidan si confían o no en la organización, lo que influenciará en la reputación corporativa.

Los beneficios de la responsabilidad social corporativa se observan en la eficiencia de la gestión y la obtención de un buen clima y compromiso laboral, el incremento en las ventas, la aceptación de productos y servicios por parte de los consumidores, entre otros; otra ventaja es la buena imagen corporativa, que propicia una mejor relación con los clientes y la fidelización de los mismos, a través de las buenas relaciones con los empleados, una disminución en conflictos con los inversores, mejoras del servicio y de la productividad con sus proveedores (Handayani \& Herwany, 2020); así pues la visión de la responsabilidad social corporativa implica extender la visión y las prácticas de la misma a aquellos stakeholders con los cuales se relaciona la empresa (Prado Lorenzo et al., 2009; Navarro García, 2008). 
Para lograr una buena gestión de la responsabilidad social corporativa en cualquier tipo de empresa que voluntariamente desee insertar dentro de sus objetivos desarrollar un rol social, se puede procurar un apoyo en instrumentos de gestión en los cuales la empresa cimienta sus objetivos estratégicos, mismos que están dentro de un conjunto de valores y principios éticos y que a su vez se reflejan en la misión y visión empresarial, y en los códigos éticos y de conducta (Poillot-Peruzzetto, 2017). Por otro lado, para la sustentabilidad ambiental hay guías como la NORMA ISO 26000 donde, a manera de guías teóricas, pueden acogerse a las definiciones, formas, lugares y medios cómo se puede comunicar a sus grupos de interés -stakeholders- el desempeño económico social y medioambiental que realizan (Gandolfi, 2012). Finalmente, las empresas pueden gestionar sus prácticas de responsabilidad social corporativa mediante una inversión socialmente responsable (Fernández Lombao \& Campos Freire, 2013), entonces a partir de estas bases no puede quedar fuera ninguna organización, incluso las de tipo comunicacional, que tienen el compromiso de informar a la comunidad e influir en la mejora de la calidad de vida, y básicamente se encuentran en el centro de una nueva economía global del sector servicios, centrándose en lo social y en la transmisión de conocimiento (Castells, 1996; Pureza \& Lee, 2020).

\section{Responsabilidad social corporativa en medios de comunicación}

En las empresas de comunicación las funciones que se desarrollan son similares a las de cualquier otra entidad dedicada a actividades mercantiles; no obstante, actúan bajo dos dimensiones de responsabilidad (Fernández Vázquez, 2012): la primera, bajo el contexto de la reciprocidad a la sociedad de los beneficios que obtiene y aplicándose de forma responsable con su entorno (González Esteban, 2007), y la segunda como un medio transmisor de información, que tiene una actuación importante, enmarcada dentro de las prácticas éticas, misma que aplicada de manera integral es el rasgo que debe definir al nuevo periodismo, en una sociedad cada vez más potencialmente abierta a la difusión y recepción de mensajes (Casasús i Guri, 2001). Sin embargo, el enfoque de la responsabilidad social corporativa en estas empresas de comunicación no debe verse desde este punto de vista ético que engloba la integridad de la información que transmiten y los compromisos con la libertad de expresión, ya que es importante considerar las actividades sociales que tienen con sus grupos de interés (Díaz Campo \& Berzosa, 2020). Por ejemplo, algunas de estas organizaciones manejan injustamente algunas funciones que afectan a sus stakeholders internos, también conocidos como empleados (Witkowska, 2016), quienes frecuentemente son sometidos a largas jornadas laborales, salarios bajos y censuras a la libertad de expresión (Valarezo González \& Marín Gutiérrez, 2013; Mahmood et al., 2020). Así mismo, en los medios de comunicación de países de Latinoamérica, como Perú, ha sido difícil que reconozcan a sus diversos stakeholders y no solo a la sociedad en la que se encuentran.

Burgos Romero (2018) admite que los medios de comunicación se han encontrado principalmente concentrados en objetivos empresariales y políticos, que influyen en los programas y los contenidos que se difunden, dejando así de lado los valores éticos y sobreponiendo aquellos principios comerciales y de marketing, que obligan a dejar de lado el aspecto social y la responsabilidad donde la premisa pasa a ser, netamente, obtener dinero comercializando sus espacios televisivos.

Pese a ello, la empresa mediática ha ido incorporando paulatinamente las prácticas de responsabilidad social en sus valores corporativos (Morales-Blanco-Steger \& Fuente-Cobo, 2018), como lo demuestran algunas empresas de comunicación en el Reino Unido, que son presionadas mediante leyes y políticas a exponer información sobre estrategias, actuaciones y riesgos, incidiendo en la transparencia del medio (Fernández Vázquez, 2012). Sin embargo, a nivel de Latinoamérica la Fundación Nuevo Periodismo Iberoamericano, con base en el estudio propuesto por Rey et al., (2008), expresa que la responsabilidad social corporativa aporta un valor agregado al negocio, que se evidencia en el mejoramiento de la eficiencia y la competitividad, y que no está sujeto solamente a desarrollar actividades de difusión de campañas sociales, sino también aspectos internos, como 
mejorar las relaciones con sus empleados. De igual modo, Sigcho Vivancho (2018) ha denotado que existen canales de televisión ecuatorianos que consolidan su fortaleza en acciones sociales mediante teletones, con el propósito de ayudar a sectores necesitados; sin embargo, la parte interna está siendo vulnerada en algunos aspectos como el recargo de horas de trabajo, temas salariales y desarrollo profesional. En definitiva, las empresas de comunicación latinoamericanas vislumbran la importancia de la responsabilidad social corporativa, pero sin establecer políticas permanentes dirigidas a sus stakeholders y menos aún a su audiencia (Orozco Toro \& Ferré Pavia, 2012).

En la actualidad, dentro del medio existen diversas formas de comunicación; sin embargo, esta investigación abordará el tema de los canales de comunicación locales, en los cuales se han podido observar ciertas acciones, en cuanto a prácticas relacionadas con responsabilidad social que están alineadas a los Artículos 84 y 85 de la Ley Orgánica de Comunicación (Asamblea Nacional, 2013), que define a los medios de comunicación privados y comunitarios, cuyo objeto está enmarcado en los servicios comunicaciones con responsabilidad social y la rentabilidad social. Por otro lado, el Artículo 92 de la misma ley regula la interrelación de estas empresas con el objetivo de establecer parámetros de equidad, respeto y responsabilidad social. Bajo esta tesitura, se plantea la hipótesis que se alinean a la ley otorgada y no se encuentran enfocados directamente en aplicar prácticas vinculadas a la responsabilidad social corporativa.

Finalmente, llegando al caso particular de la organización de comunicación televisiva cuencana Unsion TV, que fue seleccionada por la trayectoria que tiene en la ciudad, además de su credibilidad y su alto nivel de rating, se analizan a profundidad qué aspectos están considerando, bajo la perspectiva conceptual que engloba la responsabilidad social corporativa.

\section{METOdOLOGÍA}

En referencia a los objetivos general y específicos planteados ut supra, el estudio se configura desde el enfoque cuanti-cualitativo de perspectiva deductiva, fundamentado en los estudios generales de responsabilidad social corporativa analizado, desde su aplicación a la televisión local. Por una parte, el desarrollo cuantitativo de la investigación se presenta desde el alcance descriptivo, definido por Hernández Sampieri et al., (2010) como la identificación de propiedades, características y funciones del problema. En otras palabras, se busca especificar la realidad actual y la prospectiva de la situación de Unsion TV. Mientras que la aplicación cualitativa se aborda desde de investigación-acción, propendiendo la formulación de un plan de acción que genere soluciones sobre la responsabilidad social corporativa, mediante la implicación de los siguientes principios básicos: transparencia, normas, autoevaluación, gestión y consumo responsable.

En cuanto a las herramientas de recopilación de datos, se utilizaron entrevistas en profundidad y encuestas en el desarrollo estructural del análisis. En primer lugar, las entrevistas estuvieron orientadas a los stakeholders o grupos de interés internos de Unsion TV, específicamente a los empleados, quienes deberían conocer con claridad las políticas internas y la gestión que realiza esta organización, como enfoque estratégico hacia una correcta aplicación de responsabilidad social corporativa. La entrevista se elaboró en relación con las necesidades e intereses de los grupos relacionados, así como a los diferentes campos de actuación que tiene la responsabilidad social corporativa en la parte interna de la organización; dicha entrevista en profundidad fue realizada con base en doce preguntas, en las cuales se desarrollaron los criterios principales de la responsabilidad social corporativa (Carneiro, 2004). En cuanto a la confección de la entrevista, las tres preguntas iniciales se relacionaron al contexto de la responsabilidad social corporativa y a la aplicación realizada por Unsion TV; estas preguntas fueron:

1. ¿Qué es para usted la responsabilidad social corporativa? 
2. ¿Qué tan importante considera que es la gestión de la responsabilidad corporativa para la empresa?

3. ¿Conoce usted algún estándar internacional que regule las políticas de responsabilidad social corporativa?

Posteriormente, se incluyeron cinco preguntas específicas relacionadas con las acciones de Unsion TV, en cuanto a la responsabilidad social corporativa y a las dimensiones de la misma:

1. ¿Considera usted que Unsion TV está cumpliendo con la responsabilidad social corporativa?

2. ¿Hace cuánto tiempo que la empresa aplica en su trabajo responsabilidad social corporativa?

3. ¿Cuáles son los beneficios que Unsion TV obtendría mediante la aplicación de acciones que tengan que ver con la responsabilidad social corporativa?

4. Dimensión hacia la conservación ambiental. ¿¿Desarrolla Unsion TV acciones para la conservación ambiental?

5. Dimensión económica. ¿Cree usted que Unsion TV lleva a cabo políticas orientadas a la dimensión económica? ¿mantener precios bajos, ayuda a sectores vulnerables?

6. Dimensión social. ¿Cree usted que Unsion TV lleva a cabo políticas orientada a la dimensión social, es decir, mejora la calidad de vida de sus empleados, respeta los derechos humanos, tiene un código ético de conducta, financia actividades sociales y culturales y no discrimina clientes?

Las tres últimas interrogantes se direccionaron a conocer los beneficios internos que conlleva una aplicación de la responsabilidad corporativa en Unsion TV, específicamente en lo que tiene que ver con el clima laboral que perciben los empleados y la pertenencia que sienten hacia la organización, que es un factor muy importante para una mejor productividad y compromiso laboral (Barrio Fraile, 2019).

1. ¿Cómo percibe usted el clima laboral en Unsion TV?

2. ¿Qué lo incentiva a usted a laborar en Unsion TV?

3. ¿Tiene usted alguna recomendación vinculada a la responsabilidad social corporativa de Unsion TV?

A partir de allí, la Tabla 1 recopila los aspectos más importantes recogidos por las entrevistas en profundidad, siendo organizados en económicos, sociales y ambientales.

Tabla 1. Principales aspectos considerados en la entrevista

Table 1. Main topics in the interviews

\begin{tabular}{lll}
\hline Económicos & Política de precios & Ayuda a sectores vulnerables \\
\hline Sociales & $\begin{array}{l}\text { Calidad de vida de los } \\
\text { empleados }\end{array}$ & $\begin{array}{l}\text { Derechos humanos, código de ética, apoyo a } \\
\text { actividades sociales y culturales, no } \\
\text { discriminación a clientes }\end{array}$ \\
\hline Ambientales & Actividades realizadas para la conservación ambiental \\
\hline & Fuente: elaboración propia.
\end{tabular}

El desarrollo de las entrevistas tuvo lugar en las instalaciones del canal de televisión, durante tres semanas, desde el 16 de septiembre de 2019 hasta el 4 de octubre de 2019, considerando los horarios rotativos diarios del personal. Se aplicaron al total de empleados que se encontraban en roles, que fueron 25 , de los cuales el $36 \%$ corresponden a la parte administrativa, el $28 \%$ al departamento operacional del medio y el $36 \%$ al área de producción. Por otro lado, la entrevista realizada tuvo una duración de 12 a 20 minutos, las respuestas fueron registradas en una hoja de Word y grabadas bajo el consentimiento de cada trabajador, respetando el derecho de confidencialidad. 
En la última fase de la investigación se procedió a realizar las encuestas, que fueron previamente validadas por ocho expertos internacionales, catedráticos e investigadores, que ejercen sus funciones en diferentes instituciones de educación superior, como son la Universidad Rey Juan Carlos, Universidad de Nebrija, Universidad Santiago de Compostela, Universidad de Valladolid, Universidad Autónoma de Chihuahua, Universidad de Cantabria y la Universidad de Guadalajara. Se consideraron como expertos a las personas que tienen un conocimiento directo acerca de lo que se está realizando con el fin de obtener resultados aceptables (Torres-Toukoumidis \& Mäeots, 2019); además, suelen hacer una revisión crítica, sobre todo porque son conocedores de la materia.

Los expertos antes mencionados calificaron el cuestionario fundamentándose en los aspectos formales, funcionales y de contenido, en una escala del 0 al 3 siendo: 0 totalmente en desacuerdo, 1 en desacuerdo, 2 de acuerdo y 3 totalmente de acuerdo. Finalmente, se les solicitó su punto de vista sobre las preguntas de la encuesta, ya que la validación se realizó con la finalidad de conocer sus opiniones acerca de diversos aspectos y poder realizar las modificaciones correspondientes para llegar a obtener el cuestionario idóneo y definitivo; posterior a esto se procedió a la realizar las modificaciones indicadas, según los comentarios de los expertos:

Los criterios de la pregunta 3 pudieran desarrollarse más y explicar la noción de cada uno. V.gr. Para mí, para el proveedor y cliente podemos tener una definición opuesta sobre calidad de la vida laboral.

Alex Buitrago Alonso, Universidad de Valladolid.

Estos cambios se realizaron para obtener el cuestionario adecuado y así poder conocer la perspectiva que tienen los grupos de interés externos de Unsion TV con respecto a la gestión de la responsabilidad social corporativa y, de esta manera, determinar aspectos importantes, que la organización podría tener en cuenta para desarrollar acciones y políticas en torno a una eficaz aplicación de la responsabilidad social corporativa.

Luego esta herramienta fue validada y se realizó la prueba piloto en una muestra del $10 \%$ de la muestra final. Dentro de esta lista se encontraban proveedores, clientes y entes del sector público, que eran los que, en el momento de la investigación, tenían una relación activa, según las políticas de la empresa. De allí parte que la fiabilidad de esta encuesta, calculada mediante el Alpha de Cronbach, obtiene como resultado un 0,80 en cuanto a la fiabilidad, propinando así su aplicación a 50 stakeholders externos.

A partir de los ajustes producidos por la validación, la encuesta final fue formada por doce preguntas y se hizo un escalamiento de Likert de seis puntos para once interrogantes. Cabe mencionar que las escalas fueron de frecuencia donde: 1 corresponde a "casi siempre», 2 a «ocasionalmente», 3 a «raramente», 4 «nunca» y 5 «no sabe/ no contesta». Otro tipo de escala utilizada fue la de acuerdo donde: 1 es «totalmente de acuerdo», 2 «de acuerdo», 3 «indiferente», 4 «en desacuerdo», 5 «totalmente en desacuerdo». Finalmente, se incorporó la escala de veracidad, donde el encuestado podía elegir las siguientes respuestas: 1 «totalmente verdadero», 2 «parcialmente verdadero», 3 «indiferente», 4 «parcialmente falso», 5 «totalmente falso» y 6 «no sabe/ no contesta».

Existe también una pregunta de escalamiento de Likert de cinco puntos, donde 1 es «poco importante», y 5 «muy importante». En definitiva, el cuestionario se dividió en tres secciones, para las cuales se consideraron los criterios de análisis sobre responsabilidad social, emitidos por el Instituto Argentino de Responsabilidad Social y Sustentabilidad (IARSE) (2019) y Nieto Hipólito et al., (2015):

1. Valores, principios y conocimientos de la responsabilidad social de la empresa: enfocado a conocer qué piensan los stakeholders externos acerca del producto televisivo que en este caso oferta la organización; por otro lado, si las actividades que realiza, de alguna manera, están centradas en la 
ciudadanía y abordan derechos sociales. También se consideró importante conocer la perspectiva que tienen estos grupos en cuanto al grado de importancia que Unsion TV les da a varios ámbitos de la responsabilidad social corporativa, y por último el respeto y la confianza que este medio transmite, juntamente con las publicaciones abiertas que realiza acerca de sus acciones.

2. Actividades productivas y comerciales que mantienen con proveedores, clientes y agentes del sector público: orientadas a conocer el punto de vista sobre la priorización de relaciones con empresas responsables, precios y condiciones de la negociación de productos televisivos, el valor que le da Unsion TV a las opiniones, así como conocer si consideran que existe una duración de las relaciones comerciales, y la participación en actividades de responsabilidad corporativa que hayan involucrado a sus grupos de interés externos.

3. Resultados y experiencia obtenidos en los distintos tipos de relaciones que mantienen con Unsion TV: respuesta a las necesidades, sugerencias y solicitudes.

La encuesta fue aplicada vía online a los grupos de interés sugeridos por la empresa, y previamente se realizó una solicitud, vía telefónica junto con los directivos de la organización, para luego proceder satisfactoriamente.

A continuación, para analizar estos datos y obtener los resultados de la investigación acerca de la responsabilidad social corporativa de Unsion TV se utilizaron programas de Microsoft estadístico SPSS $^{\circledR}$ v. 25 y el software Atlas. Ti® ${ }^{\circledR}$.8, que sirvió de apoyo para el análisis de los datos cualitativos obtenidos en las entrevistas realizadas a los grupos de interés internos.

\section{RESULTADOS}

\section{Responsabilidad social corporativa según personal interno}

Para saber si la empresa aplica responsabilidad social corporativa y de qué manera lo hace, se procedió a realizar las entrevistas a profundidad al personal interno segmentado en administrativo, de operaciones y de producción, basándose principalmente en las dimensiones mencionadas anteriormente (Ver Tabla 1). De allí, se identificaron alrededor de 1512 frases, de las cuales 102 estaban asociadas a los principios sociales, económicos y ambientales (Ver Tabla 2).

Tabla 2. Segmentación por códigos hermenéuticos de la responsabilidad social Table 2. Classification of terms related to social responsibility

$\begin{array}{cr}\text { Código } & \text { Enraizam } \\ \text { Ambiental } & 19 \\ \text { Económico } & 28 \\ \text { Social } & 55\end{array}$

Fuente: elaboración propia a partir de Atlas.ti.

Respecto a la información recopilada en la Tabla 2, se identificó una nube de palabras representativas (ver Figura 1) con aquellas más reiteradas, en la que se omitieron adverbios y conectores, observándose que además del nombre del canal -Unsion-, también se evidencia cierta reiteración de palabras como ayuda, empleados, vulnerables, personas y sectores. 


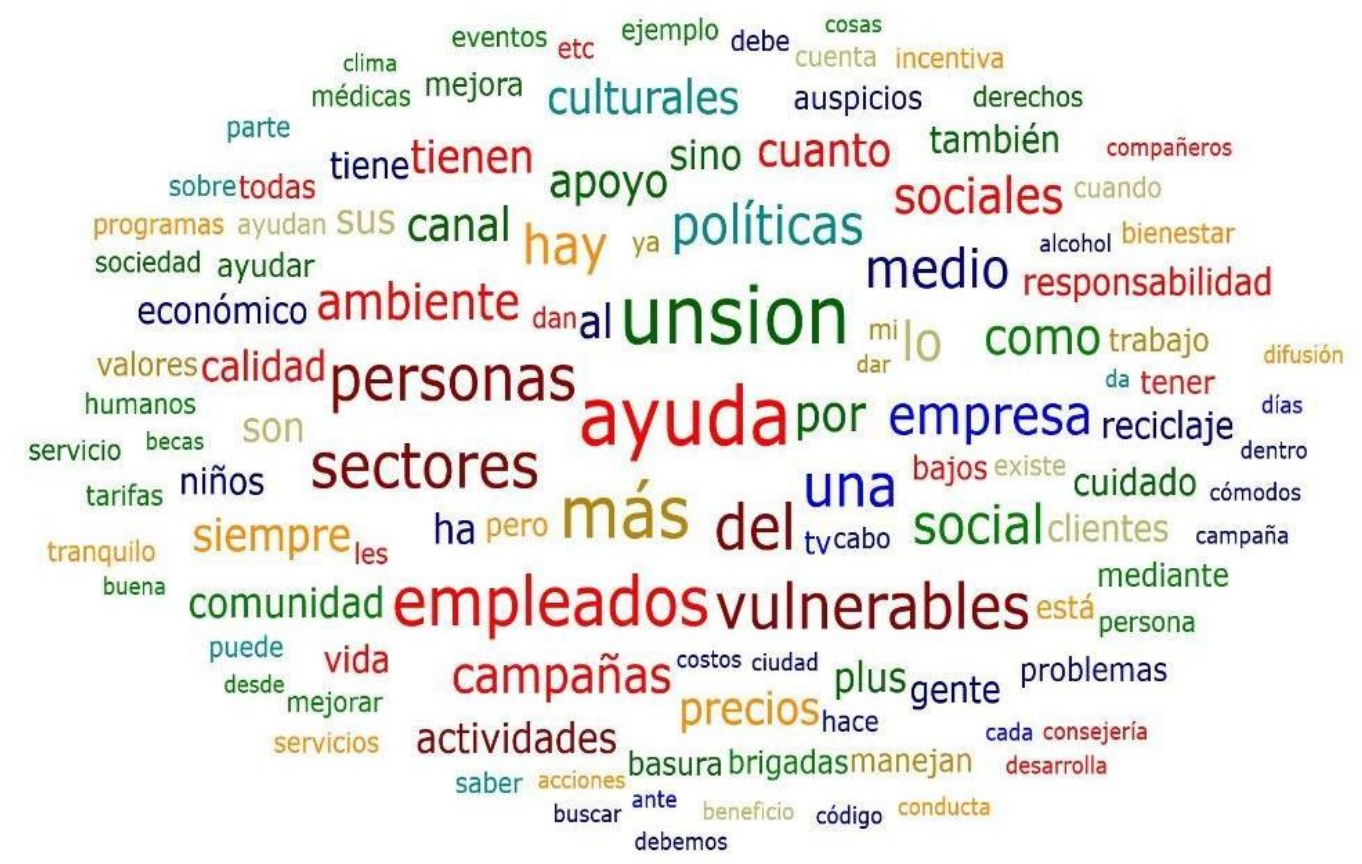

Figura 1. Nube de palabras sobre responsabilidad social corporativa según personal interno de Unsion TV Figure 1. Word cloud about corporate social responsibility according to Unsion TV employees Fuente: elaboración propia a partir de Atlas.ti.

Profundizando aún más en la información recopilada mediante las entrevistas, en la Tabla 3 consta la frecuencia y relación de las respuestas obtenidas, siendo segmentadas según departamentos: administración, operaciones y producción, alcanzando un total de 45 palabras. Vale destacar que si bien la palabra responsabilidad es la más pronunciada -55 veces-, las menos pronunciadas fueron basureros, desechos, donación, estudio, gratuita, inorgánicos, laboralmente, satisfacer, sueldos y ventas, con un total de 2 veces cada una. Complementariamente, cuando se hizo el análisis según el departamento, en aquel orientado a la administración la palabra responsabilidad fue la que más se repitió con un total de 24 veces, después políticas con 21 veces y Unsion con 19 reiteraciones. En cambio, respecto a las entrevistas de operaciones existe una triple coincidencia entre los términos políticas, medios y responsabilidad, con 14 veces cada uno, mientras que, en los encargados de producción, que constituyen principalmente el personal técnico-audiovisual, el vocablo más repetido fue Unsion con 25 veces, seguido de responsabilidad -17 veces- y precios -16 veces-. En ese tenor, la coincidencia de la palabra responsabilidad al igual que la palabra Unsion conlleva a denotar indicios de identidad y sincronía con la misión del canal. De hecho, una de las frases que contextualiza y afirma un patrón de uso de la palabra responsabilidad es la siguiente:

Una empresa debe tener en cuenta como responsabilidad el cuidado al medio ambiente, clientes y personal, más a su público externo que es la sociedad, debe haber leyes y crear acuerdos para tratar de una manera adecuada a los clientes y también al público interno. 
Tabla 3. Lista de palabras más relevantes como resultado de las entrevistas Table 3. Top words in the interviews

\begin{tabular}{|c|c|c|c|c|}
\hline Palabra & $\begin{array}{c}\text { Entrevistas } \\
\text { administración }\end{array}$ & $\begin{array}{l}\text { Entrevistas } \\
\text { operaciones }\end{array}$ & $\begin{array}{l}\text { Entrevistas } \\
\text { producción }\end{array}$ & Total \\
\hline acciones & 8 & 4 & 3 & 15 \\
\hline actividades & 8 & 7 & 10 & 25 \\
\hline ambiente & 13 & 5 & 14 & 32 \\
\hline auspicios & 5 & 1 & 4 & 10 \\
\hline basura & 4 & 2 & 2 & 8 \\
\hline basureros & 0 & 2 & 0 & 2 \\
\hline becas & 0 & 0 & 4 & 4 \\
\hline calidad & 9 & 6 & 11 & 26 \\
\hline campañas & 6 & 3 & 6 & 15 \\
\hline capacitaciones & 4 & 1 & 4 & 9 \\
\hline clientes & 10 & 12 & 15 & 37 \\
\hline comunidad & 12 & 7 & 9 & 28 \\
\hline costos & 2 & 0 & 1 & 3 \\
\hline cuidado & 3 & 1 & 2 & 6 \\
\hline culturales & 7 & 5 & 8 & 20 \\
\hline desechos & 0 & 1 & 1 & 2 \\
\hline difusión & 1 & 2 & 0 & 3 \\
\hline discriminación & 5 & 4 & 6 & 15 \\
\hline donación & 1 & 1 & 0 & 2 \\
\hline económicamente & 0 & 0 & 6 & 6 \\
\hline energía & 0 & 3 & 0 & 3 \\
\hline escuelas & 1 & 0 & 2 & 3 \\
\hline estudio & 0 & 0 & 2 & 2 \\
\hline financiamiento & 4 & 2 & 4 & 10 \\
\hline fundación & 2 & 1 & 2 & 5 \\
\hline gratuita & 1 & 0 & 1 & 2 \\
\hline inorgánicos & 0 & 2 & 0 & 2 \\
\hline laboralmente & 0 & 0 & 2 & 2 \\
\hline luz & 1 & 2 & 1 & 4 \\
\hline medio & 9 & 14 & 12 & 35 \\
\hline políticas & 21 & 14 & 14 & 49 \\
\hline precios & 13 & 7 & 16 & 36 \\
\hline proyectos & 2 & 0 & 4 & 6 \\
\hline reciclaje & 7 & 1 & 3 & 11 \\
\hline responsabilidad & 24 & 14 & 17 & 55 \\
\hline satisfacer & 1 & 0 & 1 & 2 \\
\hline servicios & 2 & 0 & 2 & 4 \\
\hline sociales & 12 & 6 & 9 & 27 \\
\hline sociedad & 6 & 5 & 6 & 17 \\
\hline sueldos & 0 & 1 & 1 & 2 \\
\hline tarifas & 2 & 0 & 2 & 4 \\
\hline Unsion & 19 & 10 & 25 & 54 \\
\hline valores & 6 & 2 & 4 & 12 \\
\hline ventas & 1 & 0 & 1 & 2 \\
\hline vulnerables & 9 & 7 & 13 & 29 \\
\hline Total & 241 & 155 & 250 & 646 \\
\hline
\end{tabular}

Fuente: elaboración propia a partir de Atlas.ti.

\section{Principio social según personal interno}

Haciendo referencia a las labores sociales, sobresalen actividades como la ayuda a sectores vulnerables «mediante la realización de brigadas médicas y psicológicas a través de Unsion Plus y el programa televisivo La Comunidad, en el cual las personas se han acercado con pedidos y Unsion TV 
se encarga de la gestión con empresas que puedan brindar ayuda; así mismo brindan ayuda con charlas motivacionales a los empleados internos y a sus familias, incentivos con becas de estudios para los hijos».

Uno de los puntos que más resaltan las personas entrevistadas es el compromiso que mantiene Unsion TV con sus empleados; sin embargo, es un tema que la empresa debe socializar con sus trabajadores, así como las políticas que emplean para mejorar la calidad de vida, ya que en muchos casos son desconocidas. Esto se evidencia, por ejemplo, en el caso del departamento de producción, donde en una de las entrevistas se manifiesta que: «Tienen manual de convivencia que ha sido muy poco socializado y no muy comunicado entre el personal, pero al mínimo conocimiento en algo se trata de aplicar». Asimismo, quienes trabajan en el área administrativa recalcan que Unsion TV, dentro de su dimensión social, se preocupa por mejorar la calidad de vida de sus trabajadores e indican que esta acción la han realizado mediante:

1. Charlas motivacionales

2. Consejería matrimonial

3. Apertura al diálogo por parte de los jefes inmediatos

4. Becas de estudio para los hijos

\section{Principio ambiental según el personal interno}

El principio ambiental fue uno de los menos mencionadas e inclusive hay quienes recalcaron que es una estrategia implementada recientemente, mediante campañas de reciclaje y conservación del agua en conjunto con la Empresa Municipal de Aseo, como es el caso del personal de administración quienes indican lo siguiente: "En la actualidad sí, los gerentes han acordado hacer por cada equipo, hay que llevar a cabo una orden para separar la basura, se usa lo que es reciclaje, eso se les entrega a las personas que venden y lo usan para poder mantenerse. La Empresa Municipal de Aseo nos ha enseñado para que podamos ayudar al medio ambiente».

Por la parte operativa indican que: «Mediante la difusión de campañas para preservar el medio ambiente, dieron cursos para reciclar y clasificar la basura». Sin embargo, la parte de producción es la que menos información tienen, en función de si la empresa realiza acciones para la conservación ambiental; hay quienes relacionan la capacidad de ser socialmente responsable con el tamaño de la empresa y consideran que esto solo lo deberían hacer las grandes empresas, e indican lo siguiente: «Es un proceso de mínimo impacto, hay pocos espacios y charlas, pero no es de gran impacto para la comunidad o colectividad».

\section{Principio económico según el personal interno}

El principio económico es un tema sobre el que únicamente tienen conocimiento quienes trabajan en los departamentos relacionados a este ámbito; por ejemplo, las entrevistas realizadas al departamento de producción, en su mayoría, indicaron que desconocían si la empresa llevaba a cabo políticas orientadas a la dimensión económica, como lo evidencia una persona quien afirmó que en relación al ámbito económico no existe socialización.

En tanto, se considera que Unsion TV debe orientarse a salvaguardar la premisa de transparencia, presentando informes respectivos a los gastos, ingresos y patrimonio a su personal interno, demostrando sus actividades, prospectiva de inversión, política de precios y ayuda a sectores vulnerables, con la finalidad de proveer claridad en los procesos de contabilidad relacionados con el desarrollo de la responsabilidad social. 


\section{Responsabilidad social corporativa según personal externo}

\section{Unsion TVy su imagen empresarial}

De acuerdo con la investigación realizada, se puede evidenciar que la organización mantiene una presencia positiva frente a los stakeholders, reflejada en el apoyo del $60 \%$ de los encuestados. Por su parte, la mayoría de los participantes consideran que la programación transmitida a la ciudadanía siempre está basada en valores positivos (90\%); cabe mencionar que estas transmisiones están formadas por programas de variedades matutinos de clasificación $A$, es decir, que pueden ser visualizados por todos los tipos de públicos.

Lo que concierne a las actividades que se realizan eventualmente por parte de Unsion TV, como son las brigadas médicas, las colonias vacacionales para los niños, las campañas de ayuda para sectores vulnerables, entre otros, se puede entrever que el $50 \%$ de encuestados están totalmente de acuerdo, y el $36 \%$ de acuerdo en que las actividades antes mencionadas están centradas en los derechos y deberes sociales, que integran a la comunidad y fomentan las buenas relaciones, que son parte de los beneficios de aplicar responsabilidad social corporativa, lo que les da una ventaja en el sector de la industria de la comunicación.

Con respecto a la ética, medio ambiente, marketing responsable, compromiso con la comunidad y calidad de vida laboral, siendo estos algunos de los ámbitos que forman parte de la responsabilidad social corporativa de una organización y que implican la participación de agentes externos como los clientes, proveedores y entes públicos, se evidencia con sus respuestas que este medio de comunicación tiene un alto sentido de responsabilidad con la comunidad, ya que el $50 \%$ considera que este compromiso es significativo, debido a que apoyan siempre campañas de empresas que realizan labor social y actividades en beneficio de la comunidad cuencana; por otro lado, en ámbitos como la ética, tan solo el $48 \%$ de los encuestados afirman que la organización le da un grado de importancia muy alto a los mismos, a excepción de la calidad de vida laboral, donde solo el $36 \%$ lo estiman como una prioridad.

En relación con la presentación pública de principios y acciones que están relacionadas con las labores, los cuales se evidencian en la Tabla 4, Unsion TV lo realiza mediante los perfiles públicos manejados a través de la web corporativa, redes sociales y medios de comunicación impresos, que formarían parte de la divulgación de la responsabilidad social corporativa que realiza la empresa, misma que se evidencia de manera totalmente afirmativa en el $62 \%$ de los encuestados.

\section{Unsion $T V$ y sus relaciones comerciales}

Por su parte, la perspectiva acerca de la priorización de relaciones con empresas que desarrollan prácticas de responsabilidad social corporativa, el $40 \%$ está totalmente de acuerdo con el apoyo a dichas acciones.

Otra de los ítems analizados demuestra que el 88\% afirma que Unsion TV presenta condiciones de negociación; es decir, se valora si el canal especifica los precios de forma transparente sobre los servicios, periodos de pago, entre otras cuestiones. Por otro lado, el $44 \%$ de los encuestados están totalmente de acuerdo con el hecho de que Unsion TV considera importante las opiniones que realizan al medio para así mejorar sus relaciones. Estas opiniones y sugerencias las toman mediante sus perfiles sociales y comentarios expuestos directamente hacia los directivos. 
Tabla 4. Respuestas relacionadas con la imagen de la empresa Table 4. Answers about the company's image

\begin{tabular}{|c|c|c|c|c|c|c|}
\hline \multirow[t]{2}{*}{$\begin{array}{l}\text { Programación } \\
\text { basada en valores }\end{array}$} & Siempre & Casi siempre & Ocasionalmente & Raramente & Nunca & $\begin{array}{c}\text { No sabe } \\
\text { / no } \\
\text { contesta }\end{array}$ \\
\hline & $60 \%$ & $30 \%$ & $6 \%$ & $4 \%$ & $0 \%$ & $0 \%$ \\
\hline \multirow{2}{*}{$\begin{array}{l}\text { Actividades } \\
\text { eventuales, cuyo } \\
\text { foco se centra en } \\
\text { la educación para } \\
\text { la ciudadanía, } \\
\text { abordando } \\
\text { derechos y } \\
\text { deberes sociales }\end{array}$} & $\begin{array}{l}\text { Totalmente } \\
\text { de acuerdo }\end{array}$ & De acuerdo & Indiferente & En desacuerdo & $\begin{array}{c}\text { Totalmente } \\
\text { en } \\
\text { desacuerdo }\end{array}$ & $\begin{array}{c}\text { No } \\
\text { sabe/ no } \\
\text { contesta }\end{array}$ \\
\hline & $50 \%$ & $36 \%$ & $12 \%$ & $2 \%$ & $0 \%$ & $0 \%$ \\
\hline $\begin{array}{l}\text { Grado de } \\
\text { importancia que } \\
\text { Unsion TV le da a } \\
\text { los ámbitos de la } \\
\text { responsabilidad } \\
\text { social corporativa }\end{array}$ & Ética & $\begin{array}{c}\text { Medio } \\
\text { ambiente }\end{array}$ & $\begin{array}{l}\text { Marketing } \\
\text { responsable }\end{array}$ & $\begin{array}{c}\text { Compromiso } \\
\text { con la } \\
\text { comunidad }\end{array}$ & \multicolumn{2}{|c|}{ Calidad de vida laboral } \\
\hline 1 Muy importante & $48 \%$ & $36 \%$ & $38 \%$ & $50 \%$ & \multicolumn{2}{|c|}{$36 \%$} \\
\hline 2 & $26 \%$ & $26 \%$ & $28 \%$ & $26 \%$ & \multicolumn{2}{|c|}{$34 \%$} \\
\hline 3 & $18 \%$ & $22 \%$ & $22 \%$ & $20 \%$ & \multicolumn{2}{|c|}{$16 \%$} \\
\hline 4 & $8 \%$ & $16 \%$ & $10 \%$ & $4 \%$ & \multicolumn{2}{|c|}{$14 \%$} \\
\hline $\begin{array}{l}5 \text { Poco } \\
\text { importante }\end{array}$ & $0 \%$ & $0 \%$ & $2 \%$ & $0 \%$ & \multicolumn{2}{|c|}{$0 \%$} \\
\hline \multirow{2}{*}{$\begin{array}{l}\text { ¿Unsion TV es } \\
\text { conocido en el } \\
\text { medio como una } \\
\text { organización } \\
\text { respetada y digna } \\
\text { de confianza? }\end{array}$} & $\begin{array}{l}\text { Totalmente } \\
\text { verdadero }\end{array}$ & $\begin{array}{l}\text { Parcialmente } \\
\text { verdadero }\end{array}$ & Indiferente & $\begin{array}{c}\text { Parcialmente } \\
\text { falso }\end{array}$ & $\begin{array}{l}\text { Totalmente } \\
\text { falso }\end{array}$ & $\begin{array}{c}\text { No } \\
\text { sabe/ no } \\
\text { contesta }\end{array}$ \\
\hline & $78 \%$ & $16 \%$ & $6 \%$ & $0 \%$ & $0 \%$ & $0 \%$ \\
\hline \multirow{2}{*}{$\begin{array}{l}\text { ¿Ha observado si } \\
\text { Unsion TV } \\
\text { presenta } \\
\text { públicamente, sus } \\
\text { principios y } \\
\text { acciones en } \\
\text { relación con sus } \\
\text { labores? }\end{array}$} & $\begin{array}{l}\text { Totalmente } \\
\text { verdadero }\end{array}$ & $\begin{array}{l}\text { Parcialmente } \\
\text { verdadero }\end{array}$ & Indiferente & $\begin{array}{l}\text { Parcialmente } \\
\text { falso }\end{array}$ & $\begin{array}{l}\text { Totalmente } \\
\text { falso }\end{array}$ & $\begin{array}{c}\text { No } \\
\text { sabe/ no } \\
\text { contesta }\end{array}$ \\
\hline & $62 \%$ & $20 \%$ & $16 \%$ & $0 \%$ & $0 \%$ & $2 \%$ \\
\hline
\end{tabular}

Referente a las relaciones duraderas y crecimiento a futuro, el $80 \%$ de los agentes externos cree que Unsion TV apoya este tipo de políticas; en otras palabras, los clientes externos tienden a mantenerse en el tiempo debido al trato personalizado, integración al medio y buenas prácticas comerciales.

Para finalizar con este apartado, si bien es cierto que estos grupos de interés evidencian algunas acciones de responsabilidad social corporativa que ejerce Unsion TV en el medio, la Tabla 5 presenta que el $24 \%$ de los encuestados expone que ocasionalmente han sido invitados a participar directamente en estas actividades; esto se hace notorio en la respuesta de la última pregunta, es decir, Unsion TV no involucra directamente a sus stakeholders externos en las acciones de responsabilidad social. 
Tabla 5. Respuestas relacionadas a las relaciones comerciales Table 5. Answers about commercial relations

\begin{tabular}{|c|c|c|c|c|c|c|}
\hline \multirow{2}{*}{$\begin{array}{l}\text { ¿Considera Ud. } \\
\text { que Unsion TV } \\
\text { prioriza sus } \\
\text { relaciones con } \\
\text { empresas que } \\
\text { desarrollan } \\
\text { prácticas de } \\
\text { responsabilidad } \\
\text { social } \\
\text { corporativa? }\end{array}$} & $\begin{array}{l}\text { Totalmente } \\
\text { de acuerdo }\end{array}$ & De acuerdo & Indiferente & En desacuerdo & $\begin{array}{c}\text { Totalmente } \\
\text { en } \\
\text { desacuerdo }\end{array}$ & $\begin{array}{c}\text { No sabe/ } \\
\text { no } \\
\text { contesta }\end{array}$ \\
\hline & $40 \%$ & $34 \%$ & $20 \%$ & $6 \%$ & $0 \%$ & $0 \%$ \\
\hline \multirow{2}{*}{$\begin{array}{l}\text { Las } \\
\text { especificaciones, } \\
\text { precios y } \\
\text { condiciones de } \\
\text { comercialización, } \\
\text { ¿están claras y } \\
\text { coinciden con los } \\
\text { servicios que se } \\
\text { ofrecen en } \\
\text { Unsion TV? }\end{array}$} & $\begin{array}{c}\text { Totalmente } \\
\text { verdadero }\end{array}$ & $\begin{array}{c}\text { Parcialmente } \\
\text { verdadero }\end{array}$ & Indiferente & $\begin{array}{l}\text { Parcialmente } \\
\text { falso }\end{array}$ & $\begin{array}{l}\text { Totalmente } \\
\text { falso }\end{array}$ & $\begin{array}{c}\text { No sabe/ } \\
\text { no } \\
\text { contesta }\end{array}$ \\
\hline & $50 \%$ & $38 \%$ & $12 \%$ & $0 \%$ & $0 \%$ & $0 \%$ \\
\hline \multirow{2}{*}{$\begin{array}{l}\text { ¿Unsion TV } \\
\text { considera que } \\
\text { sus opiniones } \\
\text { son importantes } \\
\text { para el desarrollo } \\
\text { de sus } \\
\text { actividades? }\end{array}$} & $\begin{array}{l}\text { Totalmente } \\
\text { de acuerdo }\end{array}$ & De acuerdo & Indiferente & En desacuerdo & $\begin{array}{c}\text { Totalmente } \\
\text { en } \\
\text { desacuerdo }\end{array}$ & $\begin{array}{c}\text { No sabe/ } \\
\text { no } \\
\text { contesta }\end{array}$ \\
\hline & $44 \%$ & $40 \%$ & $12 \%$ & $2 \%$ & $2 \%$ & $0 \%$ \\
\hline \multirow{2}{*}{$\begin{array}{l}\text { ¿Cree Ud. que } \\
\text { Unsion TV } \\
\text { mantiene } \\
\text { relaciones } \\
\text { comerciales } \\
\text { duraderas y } \\
\text { utiliza criterios } \\
\text { de negociación } \\
\text { que contemplan } \\
\text { su crecimiento } \\
\text { futuro? }\end{array}$} & $\begin{array}{l}\text { Totalmente } \\
\text { verdadero }\end{array}$ & $\begin{array}{l}\text { Parcialmente } \\
\text { verdadero }\end{array}$ & Indiferente & $\begin{array}{l}\text { Parcialmente } \\
\text { falso }\end{array}$ & $\begin{array}{l}\text { Totalmente } \\
\text { falso }\end{array}$ & $\begin{array}{c}\text { No sabe/ } \\
\text { no } \\
\text { contesta }\end{array}$ \\
\hline & $42 \%$ & $38 \%$ & $14 \%$ & $4 \%$ & $2 \%$ & $0 \%$ \\
\hline \multirow{2}{*}{$\begin{array}{l}\text { ¿En alguna } \\
\text { ocasión ha sido } \\
\text { invitado a } \\
\text { participar en } \\
\text { actividades de } \\
\text { responsabilidad } \\
\text { social con la } \\
\text { empresa? }\end{array}$} & Siempre & Casi siempre & Ocasionalmente & Raramente & Nunca & $\begin{array}{c}\text { No sabe/ } \\
\text { no } \\
\text { contesta }\end{array}$ \\
\hline & $24 \%$ & $16 \%$ & $24 \%$ & $20 \%$ & $12 \%$ & $4 \%$ \\
\hline
\end{tabular}

\section{Nivel de satisfacción de los stakeholders externos de Unsion TV}

En primer lugar, en esta sección se evidencia que la empresa de TV local responde directamente a las necesidades de los stakeholders de manera eficiente. Las relaciones personalizadas que se dan con estos stakeholders han permitido una cercanía y la creación de lazos que les permiten estar más comunicados y poder satisfacer cualquier requerimiento. Los grupos de interés externos se encuentran complacidos cuando el medio está consecuentemente atendiendo a las necesidades de cobertura de información, lo cual provoca el interés en invertir parte de su presupuesto público en el medio. 
Finalmente, ante las sugerencias o solicitudes que realizan los stakeholders, se deduce en la Tabla 6 que el $46 \%$ acepta que el medio de comunicación atiende de manera eficiente las necesidades como parte del procedimiento para poder atender a los grupos antes mencionados.

Tabla 6. Nivel de satisfacción

Table 6. Level of satisfaction

\begin{tabular}{|c|c|c|c|c|c|c|}
\hline $\begin{array}{c}\text { ¿Considera Ud. } \\
\text { que Unsion TV } \\
\text { corresponde de } \\
\text { manera eficiente a }\end{array}$ & Siempre & $\begin{array}{c}\text { Casi } \\
\text { siempre }\end{array}$ & Ocasionalmente & Raramente & Nunca & $\begin{array}{l}\text { No } \\
\text { sabe/ no } \\
\text { contesta }\end{array}$ \\
\hline sus necesidades? & $36 \%$ & $44 \%$ & $18 \%$ & $2 \%$ & $0 \%$ & $0 \%$ \\
\hline $\begin{array}{l}\text { ¿Considera Ud. } \\
\text { que Unsion TV } \\
\text { tiene políticas y/o } \\
\text { procedimientos de } \\
\text { relaciones para }\end{array}$ & $\begin{array}{c}\text { Totalment } \\
\mathrm{e} \\
\text { verdadero }\end{array}$ & $\begin{array}{l}\text { Parcialment } \\
\text { e verdadero }\end{array}$ & Indiferente & $\begin{array}{c}\text { Parcialment } \\
\text { e falso }\end{array}$ & $\begin{array}{c}\text { Totalment } \\
\text { e falso }\end{array}$ & $\begin{array}{c}\text { No } \\
\text { sabe/ no } \\
\text { contesta }\end{array}$ \\
\hline $\begin{array}{c}\text { responder } \\
\text { prontamente a } \\
\text { cualquier } \\
\text { sugerencia o } \\
\text { solicitud que se } \\
\text { realice? }\end{array}$ & $46 \%$ & $38 \%$ & $14 \%$ & $2 \%$ & $0 \%$ & $0 \%$ \\
\hline
\end{tabular}

\section{Plan de acción basado en estrategias genéricas hacia la responsabilidad social corporativa}

De acuerdo con la información extraída de las encuestas y entrevistas se ha determinado un plan de acción, que busca proveer de soluciones a los patrones y demandas diagnosticadas desde la visión interna como externa de Unsion TV. Por ello se construye la sistematización de un plan de acción desde cada dimensión, con el fin de proveer una serie de opciones que buscan alcanzar la optimización de la responsabilidad social corporativa.

\section{Dimensión ambiental}

Las buenas prácticas que puede realizar esta organización para mejorar la dimensión ambiental son aquellas con las que puede contribuir a reducir el riesgo ambiental en la localidad; deben de ser sencillas y útiles con el fin de trabajar juntamente con su equipo de empleados.

1. Reducción del uso de vehículos de los empleados, determinando un día a la semana para que puedan movilizarse en otros medios alternativos como bicicleta.

2. Oferta de talleres gratuitos relacionados con procesos ambientales, como pudiera ser el reciclaje o la instalación de huertas orgánicas en los hogares.

3. Difusión de informes sobre la huella de carbono, para concientizar a las personas sobre el impacto de sus acciones hacia el deterioro del medio ambiente.

4. Implementar campañas de reciclaje: conforme lo indicado en las encuestas, existe una campaña que recientemente se está socializando con la empresa pública de aseo; sin embargo, es una política que debe llevarse a cabo todo el tiempo y tener un alcance que ayude, no solo en el manejo de los residuos en Unsion TV, sino también en sus hogares.

5. Impulsar la innovación con enfoque en la eficiencia energética, con el objetivo de utilizar tecnologías que requieran una menor cantidad de uso de energía y que brinden el mismo rendimiento o la misma función; por ejemplo, el cambio a focos ahorradores, reducción de iluminación artificial e incluyendo equipos audiovisuales de carga solar.

6. Implementación de la digitalización: además de ayudar al medio ambiente a no utilizar papel, permitirá que Unsion TV incremente su habilidad empresarial y mejora de procesos por 
medio del ahorro de espacios físicos, ayuda a la rapidez de acceso a la búsqueda de archivos, además de la conservación de los mismos.

\section{Dimensión social}

Unsion TV debe tomar en cuenta aquello relacionado con el capital humano, es decir, la atención tanto a sus grupos de interés como a la sociedad cuencana en general.

1. Fortalecimiento de su departamento de ayuda social Unsion Plus que consiste en bridar ayuda psicológica y consejería gratuita, conformada por un grupo de consejeros de oración y psicólogos.

2. Se debe trabajar internamente en motivar al personal con incentivos de diferente índole, como salario, capacitación profesional y socialización de las actividades que realizan entre departamentos; esto ayudará a que el trabajador aumente su productividad.

3. Promover el comportamiento ético en Unsion TV, como un apoyo a la creación de la cultura de organización, fortaleciendo los valores y buenas prácticas, promoviendo el respeto mutuo de diversidad de religión y punto de vista político.

4. Garantizar la seguridad y la salud en el trabajo: crear una cultura preventiva en Unsion TV que sea capaz de concienciar, cambiar comportamientos y actitudes en los trabajadores, además realizar capacitaciones continuas de programas de medicina, higiene, autocuidado y seguridad industrial al trabajador.

5. Impedir la discriminación y fomentar la diversidad, mediante la elaboración de materiales orientadores de buenas prácticas que garanticen al empleado un trabajo igualitario.

\section{Dimensión económica}

Unsion TV debe estar enfocada a una acción sostenible, que oriente a la empresa a ser socialmente responsable y que valore las condiciones humanas y su realización para el bien común, entre los aspectos se podría considerar:

1. Transparencia en la socialización interna de los precios: elaboración de tablas de precios para los diferentes tipos de clientes que manejan, con el fin de mantener una información coherente para cada tipo y socializar con todos los departamentos de la empresa, y de esta manera todos puedan tener conocimiento y manejar información de manera uniforme.

2. Campañas de ayuda a sectores vulnerables con brigadas de visita puerta a puerta a los hogares con la finalidad de identificar las necesidades de las personas para posterior realizar una evaluación de los casos y determinar la ayuda que se les brindará a las familias.

3. Capacitaciones de emprendimiento para los grupos de interés con la finalidad de desarrollar la confianza entre la empresa y sus partes interesadas, así como la mejora en sus procesos para la toma de decisiones.

4. Establecer un presupuesto destinado a la ayuda económica para los trabajadores de la empresa.

\section{Imagen de la empresa}

La imagen de Unsion TV es la carta de presentación para su público objetivo, es así que se deben considerar las siguientes acciones como posibles opciones para fortalecer el concepto y la imagen que los usuarios tienen sobre la misma.

1. Buen manejo de la web corporativa: hoy en día la mayoría de los clientes se informan a través del internet, es la tarjeta de presentación de la empresa y ayuda a Unsion TV elevar su prestigio ante la comunidad y la vez aumenta el nivel de confianza con el servicio que ofrece. 
2. En cuanto a la parte directamente visual y auditiva se puede mejorar la concordancia entre los elementos como el logotipo, el nombre, la identidad corporativa, música y el slogan.

3. Trabajar en un servicio específico que puedan brindar a la comunidad con el fin de que las personas identifiquen inmediatamente.

4. Optimizar los tiempos de entrega de los servicios que brinda, estableciendo fechas límites internas para la ejecución de las tareas.

5. Campañas de street marketing además de ser consideradas de bajo presupuesto y que a su vez ayudarán a generar mayor impacto y un acercamiento directo con los stakeholders.

\section{Relaciones comerciales}

La comunicación y las buenas relaciones laborales son ejes principales que se considera que Unsion TV debería aplicar, para mantenerse como una empresa de comunicación.

Los aspectos considerados para mejorar las relaciones son:

1. Incentivar la participación directa en actividades de responsabilidad social con la empresa, mediante acuerdos de colaboración.

2. Ofrecer a los clientes presupuestos especiales.

3. Realizar estudios regulares de satisfacción, al menos una vez por año.

4. Mantener el contacto personal con todos clientes, ya que esto ayuda a construir relaciones más sólidas.

5. Llevar registros con información clave sobre los clientes, como por ejemplo datos personales, formas de contacto y actualizar la información constantemente.

\section{Nivel de satisfacción}

Es importante que Unsion TV esté al tanto del nivel de satisfacción que perciben sus grupos de interés, al mantener diferentes tipos de relaciones comerciales. Con el fin de mejorar este aspecto podría considerarse lo siguiente:

1. Mantener políticas o reglas que contemplen el establecimiento de fechas de pago para proveedores, de una forma ordenada respetando los tiempos asignados.

2. Realizar encuestas periódicas para conocer las expectativas y el grado de satisfacción de los clientes.

3. Mejorar los canales de comunicación con el cliente, es importante atender tanto sus quejas como sus sugerencias y hacerlos sentir como parte de la empresa, además de brindar respuestas rápidas en un lapso razonable de tiempo.

4. Establecer políticas y procedimientos del servicio al cliente con la finalidad de poder definir estrategias que ayuden a dar soluciones inmediatas y así mejorar su servicio.

5. Hacer uso de las redes sociales para que sirvan como canal de soporte al cliente.

\section{DISCUSIÓN}

Considerando la bibliografía analizada en el marco teórico, aquella referida a la responsabilidad social corporativa en los medios de comunicación, se observa cierta similitud en cuanto a los resultados obtenidos en esta investigación, especialmente al denotarse como un valor agregado de tipo social (Sigcho Vivancho, 2018), y por ende estableciendo diferencias con las conclusiones emitidas por Crane y Glozer (2016), quienes afirman una visión tecnocrática de los medios de comunicación hacia la responsabilidad social. Al contrario, se exhibe que la organización podría fortalecer su razón de ser trabajando en las acciones que determinan una mejor imagen, con relación a la integración de 
actividades relacionadas con la gestión de una buena responsabilidad social corporativa; no obstante, es importante mencionar que a diferencia de otros medios de comunicación nacionales e internacionales, el canal local responde de manera favorable a los intereses de sus grupos de interés internos, especialmente a sus empleados, a excepción de los principios económicos, sobre los cuales deberán mejorar su percepción mediante la promoción y difusión de los resultados obtenidos en el año contable.

Las implicaciones generadas por este estudio en el espectro práctico se resumen en proveer una visión exploratoria sobre la incursión de la responsabilidad social corporativa en el sector de la comunicación, especialmente por la ambigüedad producida por el sector al difundir una cobertura fundamentada en la responsabilidad social de contenido y omitir de su quehacer la responsabilidad social corporativa. Ambas deben cumplirse por igual y ambas deben ser prioritarias para el sector de la comunicación. De igual manera, el formato metodológico aplicado, al encontrarse validado por expertos y tener la rigurosidad científica exigida, se podrá reproducir en otros contextos.

Dentro de las futuras líneas de investigación, se podría considerar la incorporación de indicadores microeconómicos en la evaluación de la responsabilidad social corporativa, con el fin de emitir una valoración aún más cuantitativa sobre casos de televisión local. Complementariamente, un trabajo similar podría contemplar la posibilidad de realizar un análisis de la responsabilidad social corporativa del sector de la comunicación televisiva en el Ecuador, mismo que no existe al momento y que podría significar un aporte para futuros investigadores académicos y para empresarios que están interesados en tener algún tipo de relación con estas organizaciones. Finalmente se podría recomendar la aplicación del modelo en empresas de otro tipo del sector de la comunicación del Ecuador, que no sean específicamente canales de televisión, sino que emitan información mediante radio, prensa o medios modernos como empresas de publicidad digital, con la finalidad de realizar una comparativa del enfoque que tienen otras organizaciones.

\section{CONCLUSIONES}

Una vez concluido el trabajo de investigación acerca de la responsabilidad social corporativa hacia un medio de comunicación local, se evidencia que, en efecto, el medio en cuestión, y visualizado como referente general, desarrolla acciones alineadas a temas sociales, tomando en cuenta el personal externo e interno; sin embargo, también deben tenerse en cuenta que la responsabilidad social es beneficiosa para la empresa como instrumento de publicidad, especialmente coadyuvando la imagen proyectada a la comunidad donde se transmite su contenido.

En cuanto al objetivo general, se ha realizado satisfactoriamente el análisis de la responsabilidad social corporativa mediante la revisión de un marco teórico y recaudación de opiniones que englobaban las principales problematizaciones de la RSC en los medios de comunicación locales, conllevando a la emisión y aceptación de sugerencias para su aplicación e implicación eficiente en la organización. En segunda instancia, de acuerdo al primer objetivo específico, se pudo conocer desde un punto más cercano la perspectiva que tienen los grupos de interés internos con respecto a las acciones de responsabilidad social corporativa que realiza la empresa donde laboran, en la cual exigen más actividades en torno a este tema y que a la vez consideran que si se quiere tener éxito en sus operaciones comerciales, los medios locales debe dar prioridad a la realización de actividades enfocadas en este aspecto, como un proceso de su gestión y que no solamente tiene que ser visto como un aspecto exigido por las leyes, sino como una conciencia hacia el impacto causado por sus actos.

Respecto al segundo objetivo específico, se evaluó la responsabilidad social corporativa frente a los stakeholders externos que mantienen relaciones comerciales de diversa índole y que reconocen que 
los medios locales son organizaciones fiables, especialmente por su divulgación diligente y positiva en la imagen empresarial, seguido de la vinculación económica donde solicitan mayor involucramiento a eventos y pesquisas, para el mejoramiento de sinergias a través de acciones que impliquen su participación. Por último, la satisfacción general de este sector ha sido positiva, aun cuando requieran mayor atención referente a las sugerencias y solicitudes emitidas.

Finalmente, se ha desarrollado un plan de acción particularizado a los medios locales, el cual contiene una serie de recomendaciones para mejorar las actividades relacionadas con la responsabilidad social corporativa y que ha sido sugerido según los hallazgos de la investigación, con la finalidad de solventar las necesidades de los grupos de interés internos y externos; entre ellas, se debe enfatizar a nivel interno la reducción del uso de vehículos de los empleados, informes de la huella de carbono y campañas de reciclaje, promover la motivación personal mediante incentivos heterogéneos, al igual que estimular la cultura preventiva orientada a garantizar la seguridad y la salud, impulsar la transparencia en la socialización interna de los precios y, por supuesto, el apoyo a sectores vulnerables. Por su parte, a nivel externo se dispuso optimizar el uso de la web corporativa y sus redes sociales, evaluar constantemente la satisfacción de los clientes con el propósito de conocer sus necesidades inmediatas y configurar descuentos especiales a clientes con mayor fidelidad. Cabe resaltar que las sugerencias fueron aceptadas y buscan emendar la situación actual, a través de soluciones directas beneficiando colateralmente las relaciones comerciales e identidad de la empresa.

\section{REFERENCIAS}

Asamblea Nacional (2013). Ley Orgánica de Comunicación. Quito: Asamblea Nacional.

Bardos, K. S.; Ertugrul, M.; Silva Gao, L. (2020). Corporate social responsibility, product market perception, and firm value. Journal of Corporate Finance, v. 62, 101588. https://doi.org/10.1016/j.jcorpfin.2020.101588

Barrio, E.; Enrique, A. M. (2018). Responsabilidad Social Corporativa. Estudio sobre la identificación y clasificación de los stakeholders. Revista Internacional de Investigación en Comunicación, v. 17, n. 17, 90-109. https://doi.org/10.7263/adresic-017-02

Barrio Fraile, E. (2019). Responsabilidad social corporativa. De la noción a la gestión. Questiones publicitarias, v. 2, n. 24, 103-104. https://doi.org/10.5565/rev/ap.332

Burgos Romero, C. (2018). La Responsabilidad Social o sostenibilidad: Un enfoque desde el entorno y la Comunicación. Retos Revista de Ciencias de la Administración y Economía, v. 8, n. 16, 47-60. https://doi.org/10.17163/ret.n16.2018.04

Carneiro, M. (2004). La responsabilidad social corporativa interna: la nueva frontera de los recursos humanos. Editorial ESIC.

Casasús i Guri, J. M. (2001). Perspectiva ética del periodismo electrónico. Estudios Sobre El Mensaje Periodístico, n. 7, 49-55. URL

Castells, M. (1996). La era de la información: economía, sociedad y cultura. Volumen I: La sociedad red. Alianza.

Cho, E.; Tsang, A. (2020). Corporate Social Responsibility, Product Strategy, and Firm Value. AsiaPacific Journal of Financial Studies, v. 49, n. 2, 272-298. https://doi.org/10.1111/ajfs.12291 
Crane, A.; Glozer, S. (2016). Researching Corporate Social Responsibility Communication: Themes, Opportunities and Challenges. Journal of management studies, v. 53, n. 7, 1223-1252. https://doi.org/10.1111/joms.12196

Díaz Campo, J.; Berzosa, J. (2020). Responsabilidad social corporativa en empresas audiovisuales españolas. Análisis de RTVE, Atresmedia y Mediaset. Revista de Comunicación, v. 19, n. 1, 93108. https://doi.org/10.26441/RC19.1-2020-A6

Dhanesh, G. S. (2020). Who cares about organizational purpose and corporate social responsibility, and how can organizations adapt? A hypermodern perspective. Business Horizons. v. 63, n. 4 585-594. https://doi.org/10.1016/j.bushor.2020.03.011

Fernández Lombao, T.; Campos Freire, F. (2013). La Responsabilidad Social Corporativa en las radiotelevisiones públicas de Europa. Cuadernos Info, n. 33, 145-157. https://dx.doi.org/10.7764/cdi.33.530

Fernández Vázquez, J. (2012). La responsabilidad social corporativa en los principales grupos de comunicación españoles: incorporación, gestión y análisis de la información a través de sus páginas web. Correspondencias \& Análisis, n. 2, 111-127. https://doi.org/10.24265/cian.2012.n2.06

Gandolfi, V. (2012). Corporate social responsibility evaluation. Quality, supplement 2, v. 13, 1-9. URL

Gil Sánchez, G. (2018). Responsabilidad social corporativa: revisión crítica de una noción empresarial. Centro de Investigaciones Sociológicas.

Giarrizzo, V.; Maceri, S. (2019). ¿Egoístas o altruistas? Un experimento social para fomentar el comportamiento cooperativo en el mercado. Revista CEA, v. 5, n. 10, 135-150. https://doi.org/10.22430/24223182.1318

González Esteban, E. (2007). La teoría de los stakeholders Un puente para el desarrollo práctico de la ética empresarial y de la responsabilidad social corporativa. Veritas. v. 2, n. 17, 205-224. URL

Handayani, N. P.; Herwany, A. (2020). Examining the Relationship among Brand Commitment, Brand Trust, and Brand Citizenship Behavior in the Service Industry. Revista CEA, v. 6, n. 11, 13-23. https://doi.org/10.22430/24223182.1459

Hernández-Perlines, F.; Sánchez-Infantes, J. P. (2016). Análisis del Efecto de la Responsabilidad Social Empresarial en los Resultados Empresariales de las Micro, Pequeñas y Medianas Empresas (Mipymes). Revista Globalización, Competitividad y Gobernabilidad, v. 10, n. 1, 110-123. https://doi.org/10.3232/GCG.2016.V10.N1.06

Hernández Sampieri, R.; Fernández Collado, C.; Baptista Lucio, P. (2010). Metodología de la investigación. McGraw Hill.

Instituto Argentino de Responsabilidad Social y Sustentabilidad (IARSE). (2019). Reporte 2019. URL

Lizcano, J. L. (2004). ¿¿Qué es responsabilidad social corporativa? AECA.

López Salazar, A. (2013). Hacia la responsabilidad social empresarial de pequeñas empresas: caso México. Revista Internacional Administración \& Finanzas, v. 6, n. 6, 39-54. URL 
Mahmood, F.; Qadeer, F.; Abbas, Z.; Hussain, I.; Saleem, M.; Hussain, A.; Aman, J. (2020). Corporate Social Responsibility and Employees' Negative Behaviors under Abusive Supervision: A Multilevel Insight. Sustainability, v. 12, n. 7, 1-16. https://doi.org/10.3390/su12072647

Men, L. R. (2014). Internal reputation management: The impact of authentic leadership and transparent communication. Corporate Reputation Review, v. 17, n. 4, 254-272. https://doi.org/10.1057/crr.2014.14

Morales-Blanco-Steger, B.; Fuente-Cobo, C. (2018). Confianza de las audiencias en las marcas televisivas: propuesta de indicadores de responsabilidad social y reputación corporativas. El profesional de la información, v. 27, n. 3, 537-547. https://doi.org/10.3145/epi.2018.may.07

Navarro García, F. (2008). Responsabilidad social corporativa: teoría y práctica. Editorial Recenciones.

Nieto Hipólito, D. M.; Melgar Bayardo, J.; Coronel González, N. A. (2015). Cómo medir la percepción de la Responsabilidad Social Corporativa en la Industria de Dispositivos Médicos. En XX Congreso de Internacional de Contaduría, Administración e Informática. URL

Orozco Toro, J. A.; Ferré Pavia, C. (2012). La fuerza de los stakeholders en el caso de La Noria. Ciudadanía crítica y uso de redes sociales en un análisis de la reputación corporativa. Revista ICONO14 Revista Científica De Comunicación Y Tecnologías Emergentes, v. 10, n. 3, 403-424. https://doi.org/10.7195/ri14.v10i3.159

Park, Y. R.; Song, S.; Choe, S.; Baik, Y. (2015). Corporate Social Responsibility in International Business: Illustrations from Korean and Japanese Electronics MNEs in Indonesia. Journal of Business Ethics, v. 129, n. 3, 747-761. https://doi.org/10.1007/s10551-014-2212-x

Poillot-Peruzzetto, S. (2017). Le droit de la concurrence et le droit de la responsabilité sociétale des entreprises. Concurrences, n. 2, 1-3. URL

Prado Lorenzo, J. M.; García Sánchez, I. M.; Gallego-Álvarez, I. (2009). Características del consejo de administración e información en materia de Responsabilidad Social Corporativa. Revista Española De Financiación y Contabilidad, v. 38, n. 141, 107-135.

https://doi.org/10.1080/02102412.2009.10779664

Pureza, A. P.; Lee, K. H. (2020). Corporate social responsibility leadership for sustainable development: An institutional logics perspective in Brazil. Corporate Social Responsibility and Environmental Management, v. 27, n. 3, 1410-1424. https://doi.org/10.1002/csr.1894

Rey, G.; Betancourt, J.; De Paoli, M.; Abello Banfi, J.; Alonso Rozo, F. (2008). La otra cara de la libertad. La Responsabilidad Social Empresarial en medios de comunicación en América Latina. Fundación Nuevo Periodismo Iberoamericano.

Rostro Hernández, P. E.; Solís Hernández, O. (2015). Conceptualizando el diálogo entre cultura y aprendizaje organizacional. Trilogía Ciencia Tecnología Sociedad, v. 7, n. 12, 95-103.

https://doi.org/10.22430/21457778.490

Sigcho Vivancho, A. del R. (2018). La exploración de la identidad de marca televisiva: Caso tv oro-canal 2 (Trabajo de grado). Universidad Técnica de Machala, Machala, Ecuador. 
Torres-Toukoumidis, A.; Mäeots, M. (2019). Implementation of Gamification Strategies For The Enhancement Of Digital Competences. En 13th International Technology, Education and Development Conference, 9510-9518. URL

Valarezo González, K.; Marín Gutiérrez, I. (2013). La Responsabilidad Social Corporativa en empresas de comunicación en Latinoamérica. Chasqui, Revista Latinoamericana de Comunicación, n. 122, 47-52. URL

Weitzner, D.; Deutsch, Y. (2019). Why the Time Has Come to Retire Instrumental Stakeholder Theory. Academy of Management Review, v. 44, n. 3, 694-698. https://doi.org/10.5465/amr.2018.0342

Wilson, T. A. (2012). Supporting social enterprises to support vulnerable consumers: the example of community development finance institutions and financial exclusion. Journal of consumer policy, v. 35, n. 2, 197-213. https://doi.org/10.1007/s10603-011-9182-5

Witkowska, J. (2016). Społeczna odpowiedzialność biznesu - wybrane aspekty teoretyczne i empiryczne. Comparative Economic Research, v. 19, n. 1, 25-41. https://doi.org/10.1515/cer-2016-0002

Zyglidopoulos, S. C.; Carroll, C. E.; Georgiadis, A.; Siegel, D. S. (2011). Does media attention drive corporate social responsibility? Journal of Business Research, v. 65, n. 11, 1622-1627.

https://doi.org/10.1016/j.jbusres.2011.10.021 